\title{
Direct comparison of supervised and semi-supervised retraining approaches for co-adaptive BCls
}

\author{
Andreas Schwarz ${ }^{1}$. Julia Brandstetter ${ }^{1} \cdot$ Joana Pereira $^{1} \cdot$ Gernot R. Müller-Putz ${ }^{1}$ (I) \\ Received: 8 March 2019 / Accepted: 5 September 2019 / Published online: 14 September 2019 \\ (C) The Author(s) 2019
}

\begin{abstract}
For Brain-Computer interfaces (BCIs), system calibration is a lengthy but necessary process for successful operation. Coadaptive BCIs aim to shorten training and imply positive motivation to users by presenting feedback already at early stages: After just $5 \mathrm{~min}$ of gathering calibration data, the systems are able to provide feedback and engage users in a mutual learning process. In this work, we investigate whether the retraining stage of co-adaptive BCIs can be adapted to a semi-supervised concept, where only a small amount of labeled data is available and all additional data needs to be labeled by the BCI itself. The aim of the current work was to evaluate whether a semi-supervised co-adaptive BCI could successfully compete with a supervised co-adaptive BCI model. In a supporting two-class (190 trials per condition) BCI study based on motor imagery tasks, we evaluated both approaches in two separate groups of 10 participants online, while we simulated the other approach in each group offline. Our results indicate that despite the lack of true labeled data, the semi-supervised driven BCI did not perform significantly worse $(p>0.05)$ than the supervised counterpart. We believe that these findings contribute to developing BCIs for long-term use, where continuous adaptation becomes imperative for maintaining meaningful BCI performance.
\end{abstract}

Keywords Brain-computer interface $(\mathrm{BCI}) \cdot$ Motor imagery $\cdot$ Co-adaptive BCI $\cdot$ Semi-supervised learning $\cdot$ Supervised learning

\section{Introduction}

Brain-computer interfaces (BCIs) have a wide range of potential applications, whether in rehabilitation after stroke [1-3], restoring lost motor functions due to spinal cord injury [4-6], or in acquiring deeper understanding of brain functionality $[7,8]$. One of the main brain activity patterns used to design EEG-based BCIs is event-related desynchronization/ synchronization (ERD/S) [9]. ERD/S can be elicited during several mental tasks [10], being one of the most common tasks the repeated mental imagery (MI) of a movement task. One example [9] is squeezing a stress ball or plantar flexion/

A.S. and J.B. equally contributed to this study and share first authorship.

Electronic supplementary material The online version of this article (https://doi.org/10.1007/s11517-019-02047-1) contains supplementary material, which is available to authorized users.

Gernot R. Müller-Putz

gernot.mueller@tugraz.at

1 Institute of Neural Engineering, University of Technology, Stremayrgasse 16/IV, Graz 8010, Austria extension of both feet. These MI tasks lead to ERD in the alpha $(8-12 \mathrm{~Hz})$ and beta $(16-24 \mathrm{~Hz})$ frequency bands during the task, and beta ERS after movement.

In order to operate an MI-based BCI, it is typically necessary to perform a calibration step, which can be a tedious task. In this calibration step, usually dozens of repetitions for each condition are recorded to provide enough training data for preprocessing and classification algorithms [11-14], while the user does not receive any feedback. Once calibrated and operable, the BCI attempts to decode the mental imagery of the user based on its initial calibration data set, assuming stationarity of the incoming EEG signals. Hence, this type of BCI is not taking into account that EEG measurements are inherently non-stationary since the brain processes vary over time and are particularly affected by user fatigue, loss of attention, and even learning processes $[15,16]$ during the execution of the task. Aside from that, also changes in electrode impedances or other external factors can affect in real time the performance of these systems. Based on the work of Vidaurre et al. [17, 18], Faller et al. [19] developed a co-adaptive training procedure, where feedback to users was provided almost instantly: After a short data collection phase (10 trials per condition (TPC)), the system was trained and already 
provided feedback to users based on their actions. On recurrent training steps, the system retrained with all available data to improve on feedback performance. In this way, users and the $\mathrm{BCI}$ were engaged in a setup of mutual learning. We further developed the approach in [20] by integrating state-ofthe-art machine learning methods and could show in an identical experimental setup that our changes lead to a significant improvement in BCI performance by $13 \%$ on average.

Both studies could show the feasibility of early feedback presentation. Moreover, these co-adaptive systems acknowledged BCI operating users as "learners" and engaged them in a supervised mutual learning environment.

Nevertheless, these systems still rely on accurate class label information to perform model updates to effectively counter non-stationarity effects of the EEG. In a scenario of long-term, self-paced BCI use, where users do not follow a predetermined set of instructions (trials) rather than follow their causal user intention, this class label information might not be available anymore.

One approach to combine adaptive BCIs with this real-life setting is the use of unsupervised learning in adapting the classifier, which has been proposed in several studies: Hasan et al. [21] suggested an unsupervised Gaussian mixture model, which they incrementally updated in offline testing and tuning model parameters to optimally combine older and new data. Their results showed that the unsupervised adaptation performs better than no adaptation, but still worse than a classic supervised approach. Gan et al. [22] and Vidaurre et al. [23] developed methods for unsupervised adaptation of LDA classifiers by updating their mean and variance. In Gan's paper [22], the BCI system was constantly adapted after every trial by means of unsupervised clustering and subsequent updating of the LDA's mean and variance. In an online simulation of three subjects, this method proved to be effective. Vidaurre et al. [23] successfully adapted the LDA's bias in an unsupervised manner which showed no difference to adaptation using class labels in an online study. All approaches attempt to improve $\mathrm{BCI}$ performance by counteracting the nonstationarities of EEG data in long-term BCI use. Other solutions involve stopping the recurrent adaptation [24] or provide no real-time adaptation at all $[25,26]$.

Our idea to tackle this issue involves the concept of semisupervised learning: Initial calibration is performed on a comparable small set of true labeled data, but after a predefined point, only the output of the classification model is used to generate artificial labels for future data to perform recurrent trainings. Several studies have used semi-supervised learning in BCI systems, whether for a P300-based BCI speller system [27, 28] or ERD-based BCIs approaches [29-31].

In our study, we apply on the co-adaptive online BCI model presented in $[19,20,32]$ the concept of a semi-supervised retraining unit. After initial supervised calibration incorporating 50 TPC (two classes), this unit performs recurrent retrainings only using artificially generated labels. The idea is to combine the best of two worlds: On the one hand, the coadaptive approach ensures that feedback to the user is presented already after a short period of time and engages the user in a mutual learning process with the BCI. The recurrent trainings attenuate non-stationary properties of the EEG. The semisupervised concept, on the other hand, enables the BCI to become independent of labeled data - which is imperative for operating the BCI in a truly non-predetermined, selfpaced manner.

We tested this novel approach in an extended two-class MI online BCI experiment incorporating 10 healthy participants, who performed in total 190 trials per class. Additionally, we introduced three major breaks to allow potential EEG nonstationarities to take effect. Following the same experimental setup, we compare the semi-supervised concept with the supervised co-adaptive approach described in Schwarz et al. [20] on a second group of participants $(n=10)$ for comparison: We hypothesize that the semi-supervised co-adaptive approach does not perform significantly worse than the supervised co-adaptive approach, in which the classification model is trained with known true labels. We validate our hypothesis in an offline scenario where we apply each counter approach on the designated participant population.

\section{Methods}

\subsection{Participants}

Twenty healthy volunteers participated in this study. They were without any known medical conditions, and had normal or corrected to normal vision. At the beginning of the study, they were briefed about the aims of the study and gave written informed consent to participate. The study was conducted in accordance with the guidelines for ethical research according to the Declaration of Helsinki.

We assigned all participants in two groups of 10 by random selection. While group A performed the online experiment using the co-adaptive $\mathrm{BCI}$ with supervised recurrent training, similar as in [20], group B performed the online experiment using a co-adaptive BCI with semi-supervised recurrent training.

\subsection{Data acquisition}

We measured EEG with 13 active electrodes (see Fig. 1, right) covering locations over the motor cortex (FC3, FCz, FC4, C5, $\mathrm{C} 3, \mathrm{C} 1, \mathrm{Cz}, \mathrm{C} 2, \mathrm{C} 4, \mathrm{C} 6, \mathrm{CP} 3, \mathrm{CPz}, \mathrm{CP} 4)$. The reference electrode was placed on the left earlobe, ground on position $\mathrm{AFz}$. We recorded the signals using a biosignal amplifier (g.USBamp) and a g.GAMMAsys/g.LADYbird active electrode system (g.tec medical engineering $\mathrm{GmbH}$, Graz, 
Fig. 1 Experimental setup, Paradigm and electrode layout. Top left: paradigm. At second 0 , a cross appeared on the screen. At second 3 , the cue is presented followed by a 5-s imagery period. Top right: electrode setup.

Thirteen electrodes (in red) were used covering the motor cortex. Ground was positioned at $\mathrm{AFz}$ (green electrode) and for the reference we used an electrode clip at the left earlobe. Bottom: Experimental timeline. The first 10 TPC were done without feedback for gathering data to allow initial classifier calibration.

Thereafter, participants received feedback according to their actions. For the first 40, TPC model calibration was performed incorporating the supervised retraining unit (Initial). Thereafter, we changed the retraining concept for group B to the semi-supervised retraining unit, while we kept the supervised retraining concept for group A.

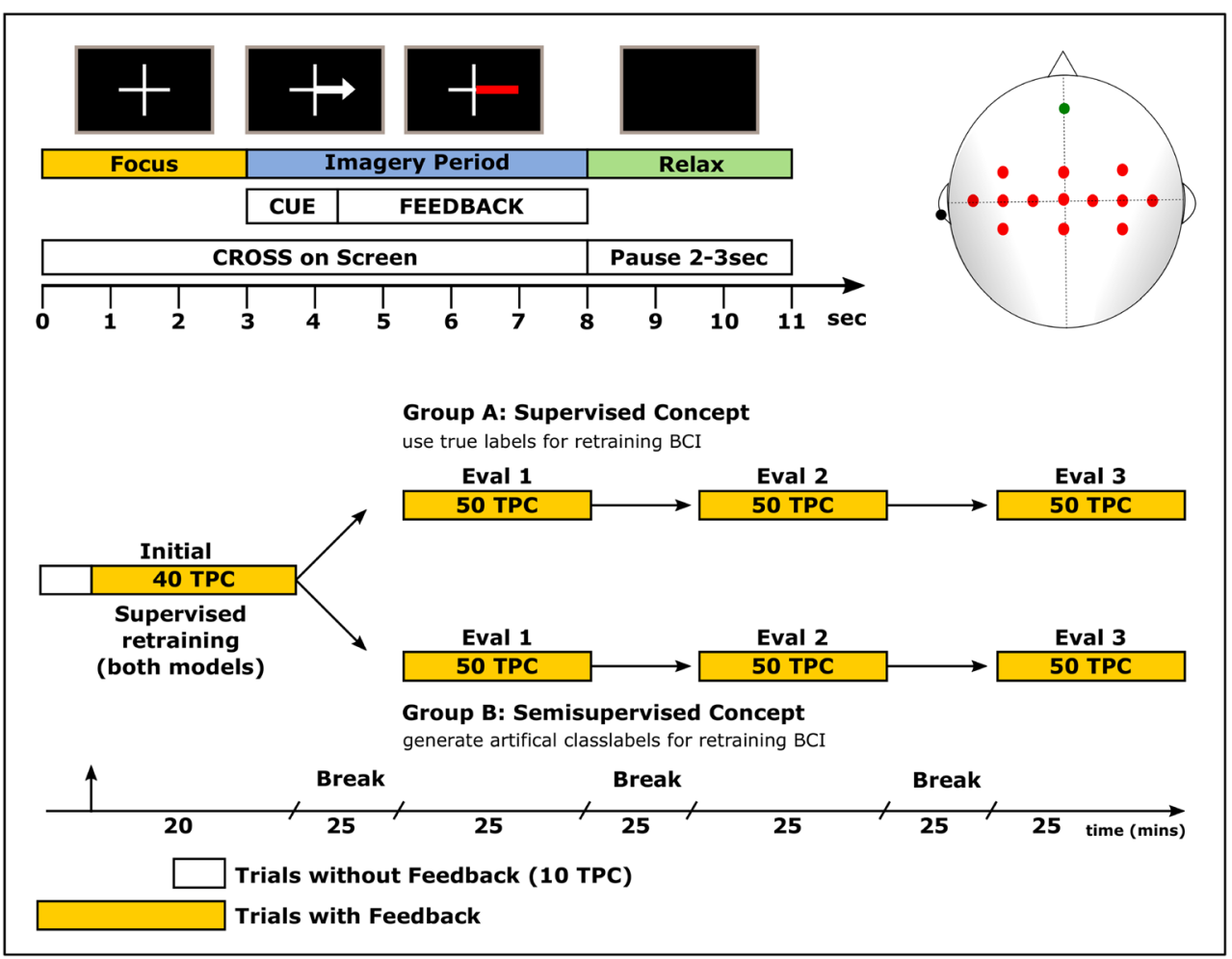

Austria). Signals were sampled at $256 \mathrm{~Hz}$ and band-pass filtered from $0.01 \mathrm{~Hz}$ to $100 \mathrm{~Hz}$ (8th-order Chebyshev filter). Power line interference was suppressed with a notch filter at $50 \mathrm{~Hz}$.

\subsection{Paradigm, experimental setup, and user feedback}

All recordings were conducted at the BCI-Lab of the Institute of Neural Engineering at the Graz University of Technology. Participants were seated in an electromagnetic and noise shielded room to facilitate consistent measurement conditions. Instructions were shown on a screen positioned at about $1 \mathrm{~m}$ in front of the participants. The paradigm was based on the standard Graz-BCI paradigm as shown in [7] (see Fig. 1, top left). At second 0 , a cross was shown on the screen. After $3 \mathrm{~s}$, an arrow pointing downwards or to the right appeared on the screen and participants were asked to imagine for $5 \mathrm{~s}$ repeated plantar flexion and extension of both feet (arrow pointing downwards) or repeated squeezing of a stress ball (arrow pointing to the right), respectively.

In the breaks $(\sim 25 \mathrm{~min})$ between the four experimental blocks, participants relaxed and watched a documentary and a TV series.

In trials in which participants were already receiving feedback according to their actions, a bar was shown at $1.25 \mathrm{~s}$ within the imagination period.

In both groups, participants performed 190 trials per class (TPC) each in 19 runs (10 TPC per run) whereas the first 10 trials were performed without feedback. After these first 10 trials and for both groups, we performed the first recurrent training and started to give feedback based on the user's actions. Feedback was presented in the form of a bar. Its length was calculated by the amount of correct classifications during the last second. If less than $50 \%$ of these classifications were correct, we showed no feedback at all since we wanted to facilitate only positive feedback [33]. We updated the feedback bar 16 times per second.

We introduced three extended breaks of about $25 \mathrm{~min}$ in our experimental design after 40, 90, and 140 TPC (see Fig. 1, bottom). Participants remained seated in front of the display and watched a nature documentary (breaks 1 and 2) and an episode of a popular TV series (break 3 ).

\subsection{Online $\mathrm{BCl}$ experiments}

The BCI system was implemented in MATLAB/ Simulink (Mathworks, Natick, MA, USA) and consisted of two main units: the online unit and the recurrent training unit. The online unit processed and classified incoming EEG data and generated the feedback in real time. In parallel, EEG data was processed by the recurrent training unit, which performed recurrent updates in the inter-trial intervals. For both participant groups, the online unit was identical, whereas the recurrent training unit performed updates incorporating a supervised 
concept (group A) or applied the proposed semisupervised concept (group B).

\subsubsection{Online unit}

We preprocessed the EEG using a filterbank [34] incorporating 15 IIR bandpass filters of order 8 . Filters were built overlapping in the $\mathrm{mu}$ and beta range $(\mu$-band $=6-8,7-9,8-10,9$ $11,10-12,11-13,12-14 \mathrm{~Hz} ; \beta$-band $=14-19,17-22,20-25$, 23-28, 26-31, 29-34, 32-37, 35-40 Hz). We combined each filter with a shrinkage regularized common spatial patterns filter (CSP) to maximize class discriminability [12]. From the resulting projections, we used the first three and the last three (CSP) projections (which contain the most discriminable information for both conditions) for further processing. Subsequently, we calculated for each remaining CSP projection (6 per filter) the signal band power using a window of $1 \mathrm{~s}$ and applied the logarithm to ensure normal distribution. In this way, we extracted 90 features for classification (15 (filters) $\times 6$ (bandpower features extracted from CSP projections)).

These features were classified incorporating a shrinkage regularized based linear discriminant analysis (sLDA) classifier $[11,35,36]$. The sLDA output was then used to adapt the length of the feedback bar as described in Section 2.3; however, for the first 10 TPC in the experiment, participants did not receive feedback since the first retraining was done after this period. After each completed trial, its prefiltered EEG correlate as well as lda scores were transferred to the designated recurrent training model.

\subsubsection{Supervised recurrent training unit (group A)}

The supervised recurrent training unit operated in parallel to the online unit and gathered all available EEG data in real time. As soon as the necessary amount of trials for retraining was accumulated (10 TPC for first training, 5 new TPC for recurrent trainings), a statistical outlier rejection was applied to all available data [19]. Here, we filtered the EEG between 3 and $35 \mathrm{~Hz}$ and rejected contaminated trials based on amplitude threshold (amplitude values exceeding $\pm 125 \mu \mathrm{V}$ ), abnormal joint probability, and abnormal kurtosis. The threshold was set to four times the standard deviation for the latter two. If five new trials passed this procedure, we accumulated them in the training datapool and performed retraining for both CSP filters and the sLDA classification model: For retraining the CSP filters, we extracted from each prefiltered trial an epoch of $3 \mathrm{~s}$ from 4.75 to $7.75 \mathrm{~s}$ with respect to the trial start [12, 37]. The sLDA classification model was trained on features extracted $5.5 \mathrm{~s}$ after trial start. Once the new models were calculated, the Online Unit was updated in the next available inter-trial interval. A detailed diagram of the supervised recurrent training unit can be found in the supplementary material Fig. S1. In the supervised approach, all true class labels were continuously used in the recurrent trainings of the system.

\subsubsection{Semi-supervised recurrent training unit (group B)}

Analogous to the supervised mode, the semi-supervised recurrent training unit assembled all available trials and continuously checked the amount of accumulated training data. Whenever five new trials per condition passed the outlier rejection, a retraining was initiated. For the first 40 TPC collected, updates happened in supervised mode, using true class labels for the training of CSP filters and the sLDA classifier (see above).

After this first calibration period, we introduced one additional selection criterion: Whenever five new TPC had passed the outlier rejection to initiate an update (accumulation of five new TPC), they were filtered according to their class probabilities. For this, we calculated for each data point within the feedback period its LDA linear score value. Thereafter, we applied the standard exponential function on each value $(z)$ and normalized it by the sum of all exponentials (see eq. 1). This so-called softmax function ensures that each component is mapped in the interval $(0,1)$, and its components add up to 1 and thus can be interpreted as (class) probabilities [38].

Softmax transformation. $K$ is the number of classes and $z$ the vector of the linear scores per classified sample

$$
\begin{aligned}
\sigma(z)_{i} & =\frac{e^{z_{i}}}{\sum_{j=1}^{K} e^{z_{j}}} R^{K} \rightarrow R^{K} \text { for } i=1 \ldots K \text { and } z \\
& =\left(z_{1} \ldots z_{K}\right) \in R^{K}
\end{aligned}
$$

For each trial, we then selected the peak class probability and calculated the first (25\%) and the third (75\%) quartile. For further retraining, we discarded all trials whose class probabilities were outside these borders and used the remaining trials for recalculating CSP filters and SLDA classification model similar as in the supervised approach.

While this additional criterion led actually to fewer retrainings of the overall system, it ensured that apart from the initial 40 TPC, the CSP and sLDA trainings utilized exclusively the system's predicted class labels. A detailed diagram of the semi-supervised recurrent training unit can be found in the supplementary material Fig. S2.

\subsection{Offline analysis}

In addition to the online $\mathrm{BCI}$ experiments, we also recreated both models offline and used the data of both groups for evaluation: for group $\mathrm{A}$, in which the supervised retraining approach was applied online, we reenacted the semi-supervised 
retraining approach offline; for group B (semi-supervised online), we reenacted the supervised approach.

Furthermore, we performed power analysis in the frequency domain using the power spectral density (PSD) estimate. For each participant, we calculated the Laplacian derivations [39] of channels C3, Cz, and C4 and used a Welch window (1 s length, no overlap) over the feedback period of each trial. Thereafter, we calculated the mean over all trials for each condition.

\subsection{Results}

Figure 2 shows PSD estimates for each subject of both groups participating in the experiment. We calculated the Laplacian derivations of channels $\mathrm{C} 3, \mathrm{Cz}$, and $\mathrm{C} 4$ and used a Welch window ( $1 \mathrm{~s}$ length, no overlap) over the feedback period of each trial to calculate the PSD. The plots show the averages per subject per condition (right hand vs. both feet).

Figure 3 depicts the online performance of both groups. We evaluated all 180 feedback trials for each subject. In general, participants of group A (supervised model) achieved lower classification performance in terms of peak accuracy (average $71.9 \%$ vs. $80.0 \%$ ) as well as mean accuracy (64.06\% vs. $72.7 \%$, calculated over the feedback period from second 4 to second 8 ) than group B (semi-supervised model). However, the performance of one participant of group B was at chance level. We used and adjusted Wald interval to determine the chance level which takes into consideration the number of trials recorded, and lies at 54.3\% (alpha =0.05) [40, 41].

Results of two-sided $t$ tests calculated over all participants' mean and peak accuracies of both groups show no significant difference (mean, $p=0.1931$; peak, $p=0.2293$ ).

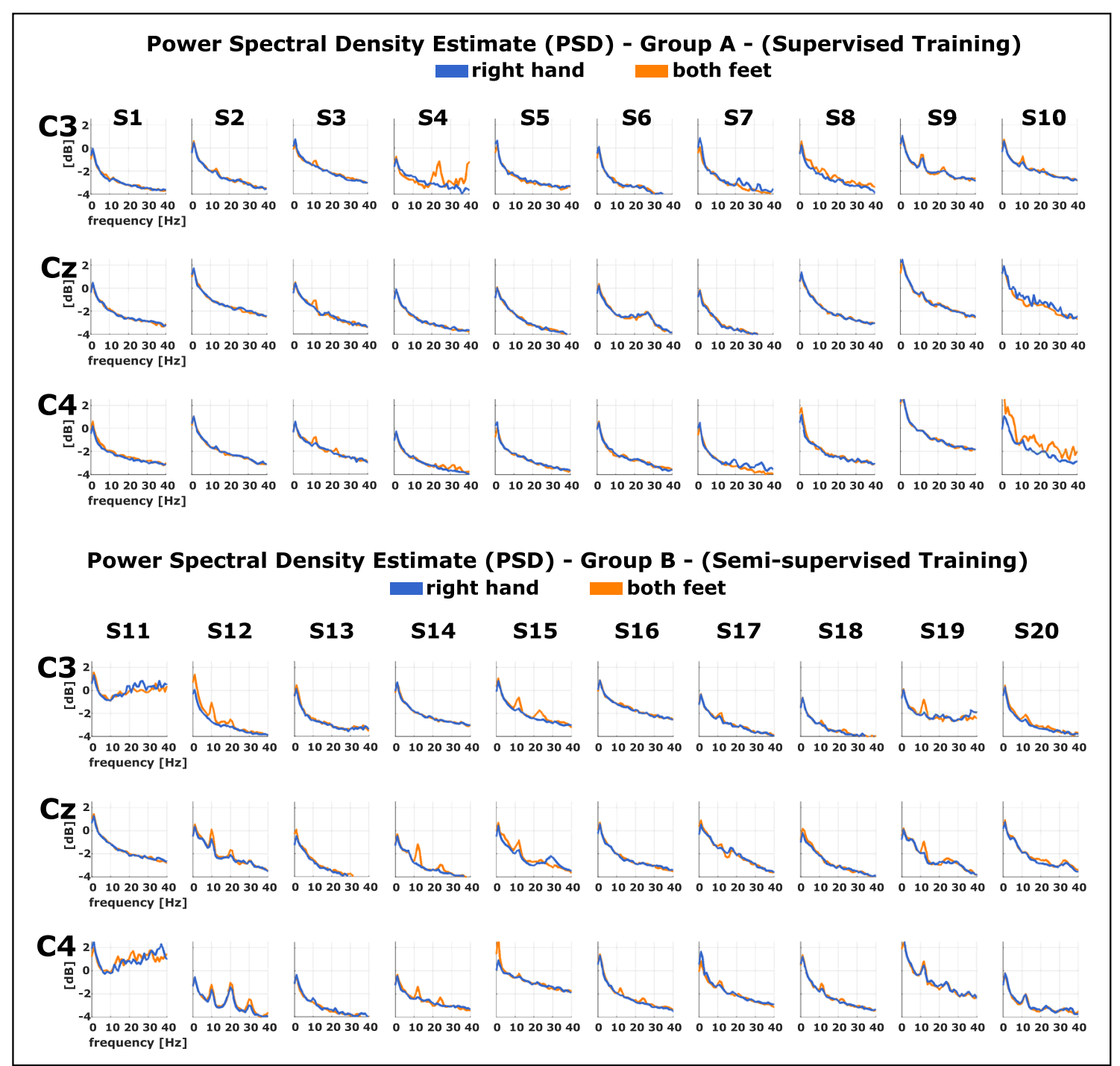

Fig. 2 Power spectral density (PSD) estimate per subject for channels C3, Cz, and C4. Abscissa shows the frequency (Hz) while the ordinate axis reflects the power $(\mathrm{dB})$ 


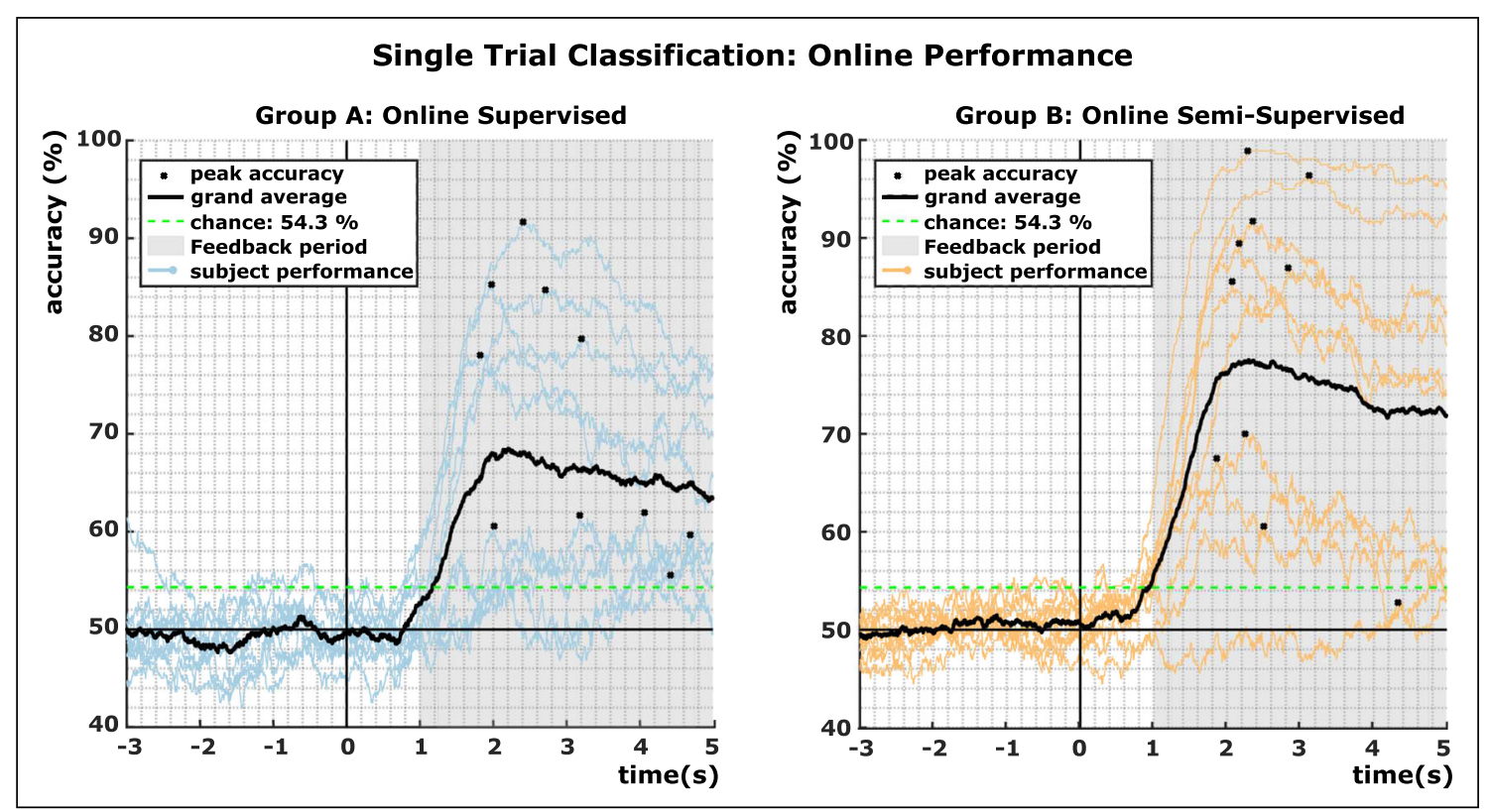

Fig. 3 Online accuracies of the single trial classification of both groups. A total of 180 feedback trials per condition were evaluated. Second 0 represent the cue onset. Colored lines show the subject-specific performance results, the black line the grand average over all subjects

We also evaluated the classification performance of both groups separately for the calibration phase (Initial $40 \mathrm{TPC}$ ), the first break (Eval 1, 50 TPC), the second break (Eval 2, 50 TPC), and after the last break (Eval 3, 50 TPC) (see Table 1). We conducted two one-way repeated measures ANOVA (one for each group) to determine whether there are significant differences in peak or mean accuracies between the different phases of the experiment. Mauchly's test for sphericity indicated that the assumption of sphericity had been violated, and therefore a correction factor to the degrees of freedom of the Fdistribution was computed using the Greenhouse-Geisser criterion. No statistically significant differences in mean (supervised, $F_{3,27}=3.165, p>0.05$; semi-supervised, $F_{3,27}=0.731$, $p>0.05$ ) or peak accuracies (supervised, $F_{3,27}=2.027, p>$ 0.05 ; semi-supervised, $F_{3,27}=0.635, p>0.05$ ) could be found for both groups. Lastly, we confirmed that there were no significant differences of mean and peak accuracies between all stages of both approaches using a mixed ANOVA (mean, $F_{3,54}=2.058, p>0.05$; peak, $F_{3,54}=1.220, p>0.05$ ).

Table 1 Intermediate performance evaluation of all 4 stages of the experiment (no significant difference between stages could be found within an approach and over all stages of both approaches)

\begin{tabular}{llllll}
\hline Stage & \multicolumn{2}{l}{ Supervised } & & \multicolumn{2}{l}{ Semi-supervised } \\
\cline { 2 - 3 } \cline { 6 - 6 } & Peak (\%) & Mean (\%) & & Peak (\%) & Mean (\%) \\
\hline Initial (1-40 TPC) & 70.5 & 60.0 & 82.2 & 74.3 \\
Eval 1 (41-90 TPC) & 76.7 & 66.5 & & 83.1 & 75.3 \\
Eval 2 (91-140 TPC) & 77.0 & 68.5 & & 83.6 & 75.8 \\
Eval 3 (141-180 TPC) & 75.6 & 66.9 & & 81.2 & 73.2 \\
\hline
\end{tabular}

Figure 4 shows the grand average for both groups of the online performance as well as the offline simulation of the counter model. The left plot shows both averages as well as the points of peak accuracy for group A. Here, the supervised approach was performed online, while we simulated the semisupervised approach offline. The course of both curves is similar, and no statistical differences in peak and mean accuracies were found (calculated over the feedback period from second 4 to second 8; Wilcoxon rank sum (WRS) test, $Z_{\text {peak }}=0.97$, $Z_{\text {mean }}=0.52, p>0.05$ ). For group B (Fig. 4, right), the semisupervised approach was performed online whereas the supervised approach was simulated offline. Again no significant differences of peak and mean accuracies were found (WRS, $\left.Z_{\text {peak }}=0.79, Z_{\text {mean }}=0.79, p>0.05\right)$. In both approaches, whether performed online or simulated offline, the supervised model performed on average better by $2 \%$.

Table 2 shows the number of recurrent trainings per approach for each subject. For the supervised approach, recurrent trainings happened considerably more often since the criterion for retraining was less strict. Finally, Table 3 shows the peak and mean accuracies for all subjects for both approaches online and offline.

\section{Discussion}

In this paper, we compared the performance of a supervised retraining approach with a semi-supervised retraining approach for $\mathrm{ERD} / \mathrm{S}$-based co-adaptive $\mathrm{BCI}$ systems. In two groups consisting of 10 participants each, we evaluated each approach online, while we 


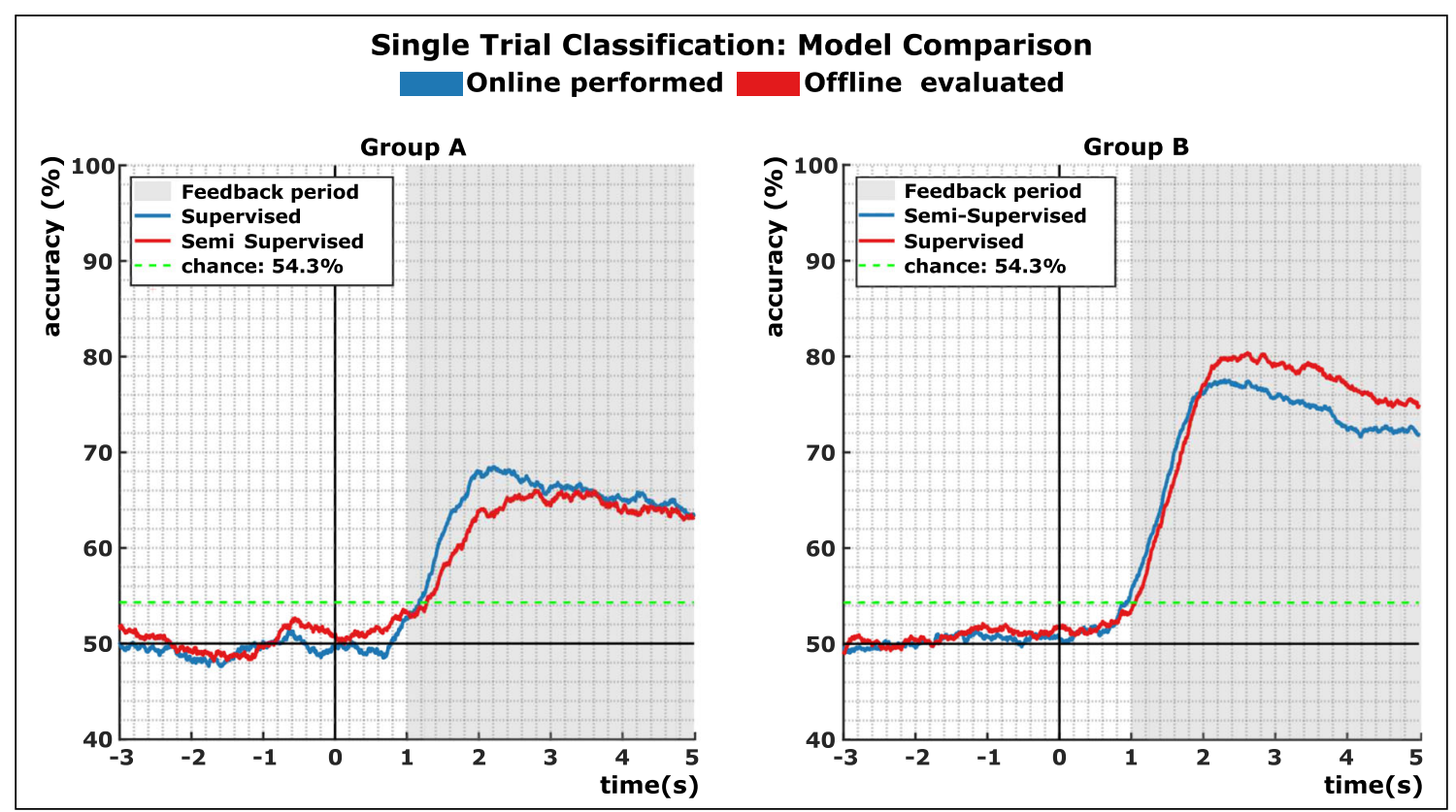

Fig. 4 Model comparison within group. The blue lines show the grand average of all subjects performed in the online part of the experiment. The red lines show the grand average of the offline simulation results of the corresponding model

simulated its counter model offline. We evaluated the accuracy for 180 feedback trials per participant. In both approaches, the grand average for online and offline analysis was significantly higher than chance with $71.9 \%$ for group $\mathrm{A}$ and $80 \%$ for group B for peak accuracies and $64.6 \%$ respective $72.7 \%$ for the mean over the feedback period. When directly comparing both approaches within the groups (where one is always simulated offline), we could show that between both approaches no significant difference, neither in peak nor mean accuracy, could be found. However, on grand average, the supervised model yielded better classification accuracies of about $2 \%$. Nevertheless, we can surmise the alternative hypothesis, namely that the semisupervised approach does not perform significantly worse than the supervised approach, can be accepted.

\subsection{Performance evaluation}

In this study, we deliberately extended the duration of our experiment not only by recording a considerably higher number of trials than in our previous co-
Table 2 Number of recurrent retrainings performed per subject

\begin{tabular}{|c|c|c|c|c|c|}
\hline \multirow[b]{2}{*}{ Number } & \multicolumn{2}{|c|}{ Group A: supervised training } & \multirow[b]{2}{*}{ Number } & \multicolumn{2}{|c|}{ Group B: semi-supervised training } \\
\hline & Online & $\begin{array}{l}\text { Offline } \\
\text { Semi-supervised }\end{array}$ & & Online & $\begin{array}{l}\text { Offline } \\
\text { Supervised }\end{array}$ \\
\hline $\mathrm{S} 1$ & 27 & 8 & S11 & 7 & 23 \\
\hline S2 & 29 & 8 & $\mathrm{~S} 12$ & 15 & 29 \\
\hline $\mathrm{S} 3$ & 31 & 10 & S13 & 13 & 31 \\
\hline S4 & 29 & 8 & S14 & 10 & 32 \\
\hline S5 & 29 & 9 & $\mathrm{~S} 15$ & 18 & 32 \\
\hline S6 & 22 & 7 & S16 & 8 & 27 \\
\hline S7 & 27 & 9 & S17 & 11 & 28 \\
\hline S8 & 25 & 12 & S18 & 13 & 27 \\
\hline S9 & 28 & 11 & S19 & 12 & 26 \\
\hline $\mathrm{S} 10$ & 23 & 8 & $\mathrm{~S} 20$ & 7 & 32 \\
\hline
\end{tabular}

For S1-S10 (group A), we simulated the semi-supervised approach offline, while we simulated the supervised approach for S11-S19 (group B). Due to the more strict selection criterion of the semi-supervised group, the number of retrainings performed in this group was considerably lower 
Table 3 Online and offline performance for both groups (mean accuracy was calculated over the feedback period from second 4 to second 8 )

\begin{tabular}{|c|c|c|c|c|c|c|c|c|c|}
\hline \multicolumn{3}{|c|}{ Group A } & \multicolumn{7}{|c|}{ Group B } \\
\hline \multirow[t]{2}{*}{ Number } & \multicolumn{2}{|c|}{$\begin{array}{c}\text { Online } \\
\text { Supervised }\end{array}$} & \multicolumn{2}{|c|}{$\begin{array}{c}\text { Offline } \\
\text { Semi-supervised }\end{array}$} & \multirow[t]{2}{*}{ Number } & \multicolumn{2}{|c|}{$\begin{array}{c}\text { Online } \\
\text { Semi-supervised }\end{array}$} & \multicolumn{2}{|c|}{$\begin{array}{c}\text { Offline } \\
\text { Supervised }\end{array}$} \\
\hline & $\begin{array}{r}\text { Peak } \\
(\%)\end{array}$ & $\begin{array}{r}\text { Mean } \\
(\%)\end{array}$ & $\begin{array}{r}\text { Peak } \\
(\%)\end{array}$ & $\begin{array}{r}\text { Mean } \\
(\%)\end{array}$ & & $\begin{array}{r}\text { Peak } \\
(\%)\end{array}$ & $\begin{array}{r}\text { Mean } \\
(\%)\end{array}$ & $\begin{array}{r}\text { Peak } \\
(\%)\end{array}$ & $\begin{array}{r}\text { Mean } \\
(\%)\end{array}$ \\
\hline S1 & 60.6 & 55.8 & 62.3 & 54.9 & S11 & 53.3 & 49.0 & 56.8 & 52.6 \\
\hline $\mathrm{S} 2$ & 78.1 & 70.2 & 72.4 & 67.6 & S12 & 96.4 & 88.6 & 96.0 & 87.5 \\
\hline $\mathrm{S} 3$ & 91.7 & 81.8 & 88.3 & 78.8 & S13 & 60.6 & 56.8 & 63.5 & 58.1 \\
\hline S4 & 59.7 & 52.8 & 55.9 & 50.0 & S14 & 86.9 & 78.9 & 88.0 & 78.7 \\
\hline S5 & 61.9 & 55.0 & 62.5 & 53.5 & S15 & 98.9 & 94.2 & 99.4 & 94.3 \\
\hline S6 & 61.7 & 55.6 & 61.8 & 55.2 & S16 & 70.0 & 60.0 & 71.6 & 66.1 \\
\hline S7 & 55.6 & 51.5 & 56.6 & 50.8 & S17 & 85.6 & 77.2 & 86.3 & 78.4 \\
\hline S8 & 79.7 & 74.2 & 76.8 & 70.5 & S18 & 91.7 & 82.6 & 91.8 & 83.2 \\
\hline S9 & 85.3 & 72.6 & 82.7 & 70.5 & S19 & 89.4 & 78.3 & 88.2 & 77.7 \\
\hline S10 & 84.7 & 76.3 & 83.5 & 75.7 & $\mathrm{~S} 20$ & 67.5 & 61.0 & 77.2 & 69.6 \\
\hline Average & 71.9 & 64.6 & 70.3 & 62.8 & & 80.0 & 72.7 & 81.9 & 74.6 \\
\hline
\end{tabular}

adaptive attempts [20, 32, 42], but also by including extended breaks. In this way, we created alternating stages of BCI control and stages where no action was needed. Our results show that, despite the extended duration of the experiment and the breaks, no significant drop in performance between stages could be determined for both supervised and semi-supervised approaches. This is an additional advantage of coadaptive BCI systems for long-term use. Moreover, it shows that the performance of the semi-supervised approach is not significantly affected by the lack of true labeling of incoming data or the extended time of use.

The number of retrainings for the semi-supervised approach decreased in comparison to the supervised approach (on average 11 vs. 27 retrainings). This was due to an additional selection criterion that excluded trials in which peak accuracies were within the first and fourth quartile of all available data for retraining: low class probabilities therefore indicated that they were close to the LDA decision boundary (e.g., within the first (below $25 \%)$ quartile) and that the classifier is more uncertain. On the contrary, high class probabilities indicated that they might not only be very distant to the LDA decision boundary but also distant to the classes' own mean distribution (e.g., within the fourth (above 75\%) quartile), making them outliers. Our results indicate that despite having a reduced number of retrainings, performance was not significantly affected. In other words, it is possible to retain all positive aspects that come with the co-adaptive approach such as shortened period where no feedback was provided or keeping the user engaged, while using only a fraction of labeled data necessary.
In direct comparison to our preceding co-adaptive work [20], lower accuracies were achieved in group A and almost equal peak and mean results in group B regardless of whether evaluated offline or online.

Both groups contained participants who scored more than $90 \%$ in online peak accuracy which indicates a considerable level of control. Only one subject in the online experiment did not achieve a classification performance above chance level. A direct comparison with other methods other than $[19,20]$ is difficult due to differences in paradigms, mental tasks, and/or experiment duration. Nevertheless, our results are within the same range as shown by the co-adaptive assessments of Acqualagna et al. in a large population study ( $n=168$, two centers, mean 72.4 and $78.3 \%$ accuracy) [43].

In comparison to the work in unsupervised adaptation of Hasan and Gan [21] and Gan et al. [22], we could show that our approach of using semi-supervised learning to adapt the classifier without true class labels works also in an online setting over an extended period of time-without a significant decrease in performance compared to the supervised adaptation. Moreover, our method of selecting classifier output labels for retrainings in an unsupervised way is relatively simple and less costly (in terms of computational load) compared to the more complex mathematical methods of unsupervised updates described in [21,23], and [22].

Of particular interest is the work from Müller et al. [15] who tackled the two-learners problem (Human $\leftrightarrow$ Machine) in a theoretical co-adaptive system simulation. In this simulation, the authors focused on the bidirectional communication between human and machine. They show that the error rates of non-adaptive BCI systems stagnate if the machine's 
learning rate is zero, which is not the case for co-adaptive BCIs. Additionally, they show that best results can be achieved by choosing controlled (mid range) learning rates for the machine, a concept which is similar to our semisupervised approach. For retraining CSP and classification models, we only incorporated "mid-range" trials with respect to their class probabilities.

\subsection{Power modulations}

We also investigated the underlying power modulations for each participant by calculating the PSD estimate for each condition. In most participants, distinct power modulations can be found, most exemplary in, e.g., S12 and S19. A decrease in power for the hand MI condition contralateral in $\mathrm{C} 3$ can be found (e.g., S12), while distinct power decrease for condition feet MI can be seen centrally at $\mathrm{Cz}$ (e.g., S19). The PSDs also show that for two participants (S10, S11), especially S10, data was contaminated with movement artifacts, most likely induced by head movements. One must therefore take this point into consideration when interpreting these participant's results since this effect could have partially contributed to their classification performance.

\subsection{Limitations and applicability for future long-term use $\mathrm{BCls}$}

In this study, we performed a between-group analysis $(n=10)$ for comparing both investigated approaches. This means that each participant only performed one approach online, while we simulated the other offline. We deliberately accepted this trade-off to avoid training effects as well as bias toward the experiment which might have influenced participant performance.

We successfully combined a co-adaptive BCI with a semisupervised recurrent retrainer unit. We could show that this novel approach is not only feasible but also works with no significant performance loss in comparison to supervised analogues [20]. We believe that this approach can be further exploited for true self-paced, long-term BCI use, where instructions are not predetermined, rather than causally determined by the BCI user. Using already executed BCI commands as "anchor" points, new trials could be extracted during runtime and added to the datapool of the retraining unit, where a class label could be artificially generated for retraining the BCI.

To further enhance reliability and stability of the BCI, it would be advisable to detect and deal with artifactual data already on site within the online BCI model rather than on the recurrent retraining unit. This could not only reduce the number of false-positive detections due to artifacts but also deny inclusion into the retraining data pool. Previous studies have already shown the benefit of detecting and dealing with artifactual EEG online [44-48].

Future investigations will show whether this approach can be scaled up by incorporating additional mental imagery classes or a rest class. Additionally, it would also be interesting to apply this concept on detection and classification of lowfrequency components triggered by complex movements such as described in [49-53].

\section{Conclusion}

In this paper, we compared a supervised with a semisupervised retraining approach for ERD-based co-adaptive BCI systems. We could show that despite the low number of labeled data (only 40 TPC of a total of 190 TPC), the semisupervised approach yielded similar performance results when compared to the supervised approach. These findings may contribute to developing BCIs for long-term use, in which continuous adaptation becomes imperative for maintaining a meaningful BCI performance.

Acknowledgments Open access funding provided by Graz University of Technology. We thank all volunteers who participated in this experiment for their effort. This work was partially funded by the EU Horizon 2020 project "MoreGrasp" (643955) and ERC Cog-2015 (681231) "Feel Your Reach."

Open Access This article is distributed under the terms of the Creative Commons Attribution 4.0 International License (http:// creativecommons.org/licenses/by/4.0/), which permits unrestricted use, distribution, and reproduction in any medium, provided you give appropriate credit to the original author(s) and the source, provide a link to the Creative Commons license, and indicate if changes were made.

\section{References}

1. Ramos-Murguialday A, Broetz D, Rea M, Läer L, Yilmaz O, Brasil FL, Liberati G, Curado MR, Garcia-Cossio E, Vyziotis A, Cho W, Agostini M, Soares E, Soekadar S, Caria A, Cohen LG, Birbaumer N (2013) Brain-machine interface in chronic stroke rehabilitation: a controlled study. Ann Neurol 74:100-108

2. Cantillo-Negrete J, Carino-Escobar RI, Carrillo-Mora P, EliasVinas D, Gutierrez-Martinez J (2018) Motor imagery-based brain-computer interface coupled to a robotic hand orthosis aimed for neurorehabilitation of stroke patients. J Healthc Eng 2018: 1624637

3. Ang KK, Guan C (2013) Brain-computer interface in stroke rehabilitation. J Comput Sci Eng 139-146. https://doi.org/10.5626/jcse. 2013.7.2.139

4. Müller-Putz GR, Scherer R, Pfurtscheller G, Rupp R (2005) EEGbased neuroprosthesis control: a step towards clinical practice. Neurosci Lett 382:169-174

5. Rohm M, Schneiders M, Müller C, Kreilinger A, Kaiser V, MüllerPutz GR, et al. (2013) Hybrid brain-computer interfaces and hybrid neuroprostheses for restoration of upper limb functions in 
individuals with high-level spinal cord injury. Artif Intell Med. pp. 133-142. doi:https://doi.org/10.1016/j.artmed.2013.07.004

6. Rupp R, Rohm M, Schneiders M (2018) Brain-computer interfaces for control of upper extremity neuroprostheses in individuals with high spinal cord injury [Internet]. Biomedical Eng. pp. 809-836. doi:https://doi.org/10.4018/978-1-5225-3158-6.ch036

7. Pfurtscheller G, Neuper C (2001) Motor imagery and direct braincomputer communication. Proc IEEE 89:1123-1134

8. Müller-Putz GR, Schwarz A, Pereira J, Ofner P (2016) From classic motor imagery to complex movement intention decoding: the noninvasive Graz-BCI approach. Prog Brain Res 228:39-70

9. Pfurtscheller G, Lopes da Silva FH (1999) Event-related EEG/ MEG synchronization and desynchronization: basic principles. Clin Neurophysiol 110:1842-1857

10. Friedrich EVC, Neuper C, Scherer R (2013) Whatever works: a systematic user-centered training protocol to optimize braincomputer interfacing individually. PLoS One 8:e76214

11. Blankertz B, Lemm S, Treder M, Haufe S, Müller K-R (2011) Single-trial analysis and classification of ERP components- a tutorial. Neuroimage. 56:814-825

12. Ramoser H, Müller-Gerking J, Pfurtscheller G (2000) Optimal spatial filtering of single trial EEG during imagined hand movement. IEEE Trans Rehabil Eng 8:441-446

13. Lotte F, Bougrain L, Cichocki A, Clerc M, Congedo M, Rakotomamonjy A, Yger F (2018) A review of classification algorithms for EEG-based brain-computer interfaces: a 10 year update. J Neural Eng 15:031005

14. Steyrl D, Scherer R, Faller J, Müller-Putz GR (2016) Random forests in non-invasive sensorimotor rhythm brain-computer interfaces: a practical and convenient non-linear classifier. Biomed Tech 61:77-86

15. Müller JS, Vidaurre C, Schreuder M, Meinecke FC, von Bünau P, Müller K-R (2017) A mathematical model for the two-learners problem. J Neural Eng 14:036005

16. Grosse-Wentrup M, Schölkopf B (2013) A review of performance variations in SMR-based brain-computer interfaces (BCIs). SpringerBriefs in Electrical and Computer Engineering. pp. 3951. doi:https://doi.org/10.1007/978-3-642-36083-1_5

17. Vidaurre C, Sannelli C, Müller K-R, Blankertz B (2011) Coadaptive calibration to improve BCI efficiency. J Neural Eng 8: 025009

18. Vidaurre C, Sannelli C, Müller K-R, Blankertz B (2010) Machinelearning based co-adaptive calibration: a perspective to fight $\mathrm{BCI}$ illiteracy. Lect Notes Comput Sci. pp. 413-420

19. Faller J, Vidaurre C, Solis-Escalante T, Neuper C, Scherer R (2012) Autocalibration and recurrent adaptation: towards a plug and play online ERD-BCI. IEEE Trans Neural Syst Rehabil Eng 20:313-319

20. Schwarz A, Scherer R, Steyrl D, Faller J, Muller-Putz GR (2015) A co-adaptive sensory motor rhythms brain-computer Interface based on common spatial patterns and random forest. Conf Proc IEEE Eng Med Biol Soc 2015:1049-1052

21. Hasan BAS, Gan JQ (2009) Unsupervised adaptive GMM for BCI [Internet]. 2009 4th International IEEE/EMBS Conference on Neural Engineering. doi:https://doi.org/10.1109/ner.2009.5109291

22. Gan JQ. Self-adapting BCI based on unsupervised learning cswww. essex.ac.uk. Available: http://cswww.essex.ac.uk/Research/BCIs/ BCI06 GAN2.pdf

23. Vidaurre C, Kawanabe M, von Bünau P, Blankertz B, Müller KR (2011) Toward unsupervised adaptation of LDA for braincomputer interfaces. IEEE Trans Biomed Eng 58:587-597

24. Cunha JD, Scherer R (2018) Are online co-adaptive sensorimotor rhythm brain-computer interface training paradigms effective? 2018 International Conference on Cyberworlds (CW). doi:https:// doi.org/10.1109/cw.2018.00081

25. Schwarz A, Steyrl D, Muller-Putz GR (2016) Brain-computer interface adaptation for an end user to compete in the Cybathlon.
2016 IEEE International Conference on Systems, Man, and Cybernetics (SMC). doi:https://doi.org/10.1109/smc.2016. 7844499

26. Millán JDR, Del R. Millán J (2010) Combining brain-computer interfaces and assistive technologies: state-of-the-art and challenges. Front Neurosci;1. doi:https://doi.org/10.3389/fnins.2010. 00161

27. Li Y, Guan C, Li H, Chin Z (2008) A self-training semi-supervised SVM algorithm and its application in an EEG-based brain computer interface speller system. Pattern Recogn Lett 29:1285-1294

28. Gu Z, Yu Z, Shen Z, Li Y (2013) An online semi-supervised braincomputer interface. IEEE Trans Biomed Eng 60:2614-2623

29. Nicolas-Alonso LF, Corralejo R, Gomez-Pilar J, Álvarez D, Hornero R (2015) Adaptive semi-supervised classification to reduce intersession non-stationarity in multiclass motor imagerybased brain-computer interfaces. Neurocomputing. 159:186-196

30. Ang KK, Chin ZY, Zhang H, Guan C (2011) Filter Bank Common Spatial Pattern (FBCSP) algorithm using online adaptive and semisupervised learning. The 2011 International Joint Conference on Neural Networks. doi:https://doi.org/10.1109/ijcnn.2011.6033248

31. Bamdadian A, Guan C, Ang KK, Xu J (2012) Online semisupervised learning with $\mathrm{KL}$ distance weighting for motor imagery-based BCI. Conf Proc IEEE Eng Med Biol Soc 2012: 2732-2735

32. Faller J, Scherer R, Costa U, Opisso E, Medina J, Müller-Putz GR (2014) A co-adaptive brain-computer interface for end users with severe motor impairment. PLoS One 9:e101168

33. Barbero A, Grosse-Wentrup M (2010) Biased feedback in braincomputer interfaces. J Neuroeng Rehabil 7:34

34. Ang KK, Chin ZY, Wang C, Guan C, Zhang H (2012) Filter bank common spatial pattern algorithm on BCI competition IV datasets 2a and 2b. Front Neurosci;6. doi:https://doi.org/10.3389/fnins. 2012.00039

35. Ledoit O, Wolf M (2004) A well-conditioned estimator for largedimensional covariance matrices. J Multivar Anal. pp. 365-411. doi:https://doi.org/10.1016/s0047-259x(03)00096-4

36. Steyrl D, Scherer R, Förstner O, Müller-Putz GR (2014) Motor imagery brain-computer interfaces: random forests vs regularized LDA - non-linear beats linear. Proceedings of the 6th International Brain-Computer Interface Conference. pp. 241-244

37. Scherer R, Pfurtscheller G, Neuper C (2008) Motor imagery induced changes in oscillatory EEG components: speed vs. accuracy. Proceedings of the 4th International Brain-Computer Interface Workshop and Training Course 2008. Verlag der Technischen Universität Graz; pp. 186-189

38. Bishop CM (2016) Pattern recognition and machine learning. Springer

39. Hjorth B (1975) An on-line transformation of EEG scalp potentials into orthogonal source derivations. Electroencephalogr Clin Neurophysiol 39:526-530

40. Billinger M, Daly I, Kaiser V, Jin J, Allison B, Mueller-Putz G (2013) Is it significant? Guidelines for reporting BCI performance. In: Allison BZ, Dunne S, Leeb R, Millan JDR, Nijholt A (eds) Towards Practical Brain-Computer Interfaces. Springer, pp 333354

41. Müller-Putz GR, Scherer R, Brunner C, Leeb R, Pfurtscheller G (2008) Better than random? A closer look on BCI results. International Journal on Biomagnetism 10:5

42. Faller J, Scherer R, Friedrich EVC, Costa U, Opisso E, Medina J, Müller-Putz GR (2014) Non motor tasks improve adaptive braincomputer interface performance in users with severe motor impairment. Front Neurosci;8. doi:https://doi.org/10.3389/fnins.2014. 00320

43. Acqualagna L, Botrel L, Vidaurre C, Kübler A, Blankertz B (2016) Large-scale assessment of a fully automatic co-adaptive motor imagery-based brain computer interface. PLoS One 11:e0148886 
44. Daly I, Scherer R, Billinger M, Müller-Putz G (2015) FORCe: fully online and automated artifact removal for brain-computer interfacing. IEEE Trans Neural Syst Rehabil Eng 23:725-736

45. Daly I, Billinger M, Laparra-Hernández J, Aloise F, García ML, Faller J, Scherer R, Müller-Putz G (2013) On the control of brain-computer interfaces by users with cerebral palsy. Clin Neurophysiol 124:1787-1797

46. Scherer R, Schloegl A, Lee F, Bischof H, Jansa J, Pfurtscheller G (2007) The self-paced Graz brain-computer interface: methods and applications. Comput Intell Neurosci; 79826

47. Qi W (2017) Algorithms benchmarking for removing EOG artifacts in brain computer interface. Clust Comput. https://doi.org/10.1007/ s10586-017-1157-0

48. Fatourechi M, Bashashati A, Ward RK, Birch GE (2007) EMG and EOG artifacts in brain computer interface systems: a survey. Clin Neurophysiol 118:480-494

49. Schwarz A, Ofner P, Pereira J, Sburlea AI, Müller-Putz GR (2018) Decoding natural reach-and-grasp actions from human EEG. J Neural Eng 15:016005

50. Pereira J, Ofner P, Schwarz A, Sburlea AI, Müller-Putz GR (2017) EEG neural correlates of goal-directed movement intention. Neuroimage. 149:129-140

51. Omedes J, Schwarz A, Müller-Putz GR, Montesano L (2018) Factors that affect error potentials during a grasping task: toward a hybrid natural movement decoding BCI. J Neural Eng 15:046023

52. Ofner P, Schwarz A, Pereira J, Wyss D, Wildburger R, Müller-Putz GR (2019) Attempted arm and hand movements can be decoded from low-frequency EEG from persons with spinal cord injury. Sci Rep. https://doi.org/10.1038/s41598-019-43594-9

53. Iturrate I, Chavarriaga R, Pereira M, Zhang H, Corbet $\mathrm{T}$, Leeb R, Millán JR (2018) Human EEG reveals distinct neural correlates of power and precision grasping types. Neuroimage. 181:635-644

Publisher's note Springer Nature remains neutral with regard to jurisdictional claims in published maps and institutional affiliations.

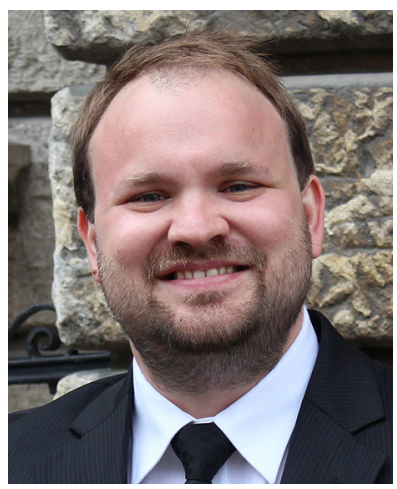

Andreas Schwarz is a research assistant at the Institute of Neural Engineering (INE) at Graz University of Technology. His current research focus lies on EEG-based decoding of grasping for brain-computer interface control.

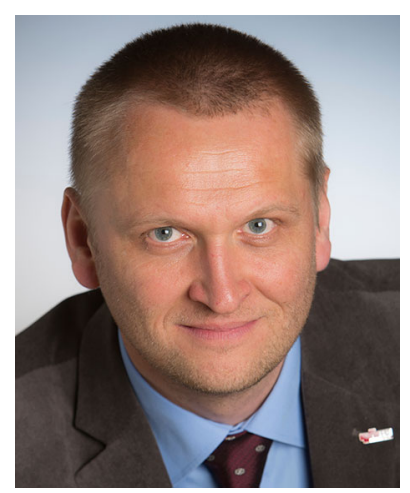

Julia Brandstetter graduated in 2017 in Biomedical Engineering at Graz University of Technology. Currently, she works as an analytics engineer at Meon Medical Solutions, Graz.

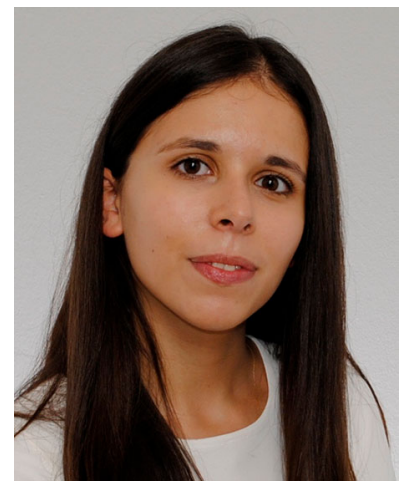

Joana Pereira is a research assistant at the Institute of Neural Engineering (INE) at Graz University of Technology. Her research focuses on investigating goal-directed movement planning and detecting movement intentions using EEG.

Gernot R. Müller-Putz, full professor, is head of the Institute of Neural Engineering and its associated BCI-Lab. Recently, he coordinated the EU H2020 Project MoreGrasp and was awarded with an ERC Consolidator Grant "Feel your Reach" in 2015. 